It was thought that arrhythmias and tachycardias other than sinus tachycardia were uncommon in pulmonary disease. It is clear from intensive study of pulmonary disease and particularly pulmonary heart disease that atrial arrhythmias and tachycardias are more common than previously thought (Corazza and Pastor, 1958; Suppa, Martini, and Gazzi, 1967). It is generally agreed that atrial fibrillation is rare in pulmonary heart disease, and that ventricular disturbances are less common than atrial disturbances. To study further the types and possible explanations of arrhythmias and tachycardias in chronic pulmonary disease we have looked at the findings in 102 cases with important disorders of rhythm and rate. These were selected from the admissions over a three-year period to one hospital. The admissions of chronic pulmonary disease cases over the three-year period number 1482 and the arrhythmias and tachycardias form 6.9 per cent of these.

All patients were in hospital for more than 5 days. All had more than one cardiogram recorded, and all were either admitted on more than one occasion or were followed as out-patients for a long period. Seven were below the age of 50 years and 55 were above 59 years of age. There were 41 deaths, with necropsy findings recorded in 30 of these. Chronic bronchitis was the main diagnosis in 53 cases and was additional to pneumoconiosis in 37 others. There were 12 cases of asthma. Of the 37 cases of coalworkers' pneumoconiosis and bronchitis, 14 had simple pneumoconiosis, 3 had early changes of progressive massive fibrosis, and 20 had advanced progressive massive fibrosis. There were 12 cases

Received January 8, 1969.

$\star$ The studies of the pulmonary circulation of which this is a part are supported by grants from the British Heart Foundation and the United Cardiff Hospitals, and we gratefully acknowledge these. of significant coronary artery disease which had not been recognized clinically-all in the bronchitis group.

In 55 of the patients an acute chest infection had been the reason for admission, and others were found to have persisting infection when in hospital. Not one patient was admitted to hospital because of the tachycardia or arrhythmia alone. In 76 of the patients there was severely reduced ventilatory capacity, $\mathrm{FEV}_{1.0}$ below one litre and $\mathrm{FEV}_{1.0}$ : FVC ratio below 50 per cent. Fourteen had $\mathrm{FEV}_{1.0}$ between 1.0 and 1.4 litre, 5 had FEV $_{1.0} 1.5-1.9$ litre, and in 7 it was not recorded. The length of history was hard to establish, but in all except 15 patients it was regarded as more than 5 years, and in 58 cases the history was clearly documented over 10 years.

Sinus tachycardia is a frequent accompaniment of any additional disturbance in chronic pulmonary disease, and every patient in this series had sinus tachycardia of 100 beats a minute or more at some time, and this was not analysed further unless there were cardiographic changes of arrhythmia or a heart rate over 140 a minute.

\section{Atrial TACHYCARDia}

Atrial tachycardia was the commonest finding and it was present in 32 cases. All but 3 had chronic bronchitis; 13 also had coalworkers' pneumoconiosis. Four were later found to have coronary artery disease of significant degree. The 3 without chronic bronchitis had acute bronchitis and asthma.

Fourteen of the 32 patients were admitted with acute chest infection and a further 5 had respiratory failure, the result of infection. All the patients had 491 
measured the oxygen saturation was much reduced. In 14 of the 32 the cardiographic pattern was that of classical right ventricular hypertrophy, and in 14 others there were cardiographic features of pulmonary heart disease. One showed a pattern of left ventricular hypertrophy and one of combined hypertrophy.

As far as could be judged, digitalis did not play a part in the causation of the atrial tachycardia in any case, and in only 8 patients was a digitalis effect evident at any time during the admission.

Four of the cardiograms had right bundle-branch block pattern associated in all 4 with right ventricular hypertrophy. There was no instance of a low serum potassium in this group. A raised blood urea is not uncommon in pulmonary heart disease, and 12 patients in this group had values for blood urea between $40 \mathrm{mg}$. and $79 \mathrm{mg} . / 100 \mathrm{ml}$. and $4 \mathrm{had}$ values over $80 \mathrm{mg}$., but any part played by this blood urea increase in the occurrence of atrial tachycardia could not be assessed.

An interesting finding at necropsy was the presence of thrombosis of a major branch of the pulmonary arterial tree in 4 of these patients, and there was some evidence in the clinical record that the thrombotic happening was related to the episode of atrial tachycardia in that the clinical state of the patient worsened suddenly with hypotension just before the onset of the tachycardia.

Study of the cardiograms recorded in the days before an episode of tachycardia showed disturbances that might be warning signs. In 7 there were atrial ectopic beats and in 5 there was marked variation in the $\mathbf{P}$ waves. In 2 there was a short $P-R$ interval, and more strikingly 10 of the 32 had a long Q-T interval.

Atrial tachycardia is certainly not uncommon in pulmonary disease, occurring in cases with pulmonary heart disease, but occasionally in exacerbations of pulmonary disease alone due to a respiratory infection or respiratory failure. Some evidence in previous work suggested that atrial tachycardias indicated a poor prognosis (Rees, Thomas, and Rossiter, 1964). This supposition is supported in this study.

\section{Atrial Flutter}

In this series atrial flutter appeared in 2 patients, and both had a significant degree of coronary artery disease. It is not possible to say whether the coronary artery disease played a part in producing atrial flutter. The arrhythmia was associated with severe failure in both patients, heart failure being present before the atrial flutter appeared and worsening greatly with its onset. In other work of ours we have seen patients with atrial flutter and pure pulmonary heart disease, but this was rare.

\section{Atrial Fibrillation}

This was present in 6 patients. Two of these were later shown to have coronary artery disease of significant degree, and another at necropsy had a diffuse myocardial fibrosis. The other 3 patients had severe pulmonary heart disease and one of them was in respiratory failure. It seems that atrial fibrillation does appear in pure pulmonary heart disease, but its occurrence is rare and it is important to look for another predisposing factor when it appears.

\section{AV Nodal Rhythm}

This abnormal rhythm was seen in 10 cases. All the patients were severely ill and 8 of them died later. Two were shown to have pulmonary arterial branch thrombosis and another had pulmonary infarction due to embolism. Two of the patients subsequently had other arrhythmias. All had heart rates over 100 a minute at some time. In one of the patients there had been persistent nodal rhythm with a normal heart rate for 3 years, an unusual finding. Serum potassium in these patients was within normal limits. On the whole these patients fared badly.

There were 7 instances of AV nodal ectopic beats. In 3 of these there followed an atrial tachycardia.

Reference must be made to 2 patients with nodal $P$ waves and normal $P-R$ interval. It is of interest that in one of these cases the $P$ wave amplitude was $2.5 \mathrm{~mm}$. in one standard lead, giving inverted $P$ waves comparable to "pulmonary $P$ waves". This was an unusual finding because a cardiogram on another day showed normal sinus rhythm without "pulmonary $P$ waves".

\section{WANDERING PACEMAKER}

This was considered to be present when there was variation of the form of the $P$ wave, with some variation in the $R-R$ intervals but with no set pattern; there were 18 examples. Four were regarded as wandering of the pacemaker in the sinoatrial node, all 4 were severely ill and hypoxic at the time, but subsequent cardiograms showed normal sinus $P$ waves. In 14 cases $P$ waves and $R-R$ intervals suggested variation of the focus through the atria. The cardiographic patterns in these patients were those of right ventricular hypertrophy or of pulmonary heart disease. All the patients with a wandering pacemaker presented at some other time with abnormal rhythms or tachy- 
cardias. It does seem that this instability of the origin of the $\mathrm{P}$ wave occurs with severe hypoxia and pulmonary hypertension.

\section{VARYING $P$ WAVES}

It was a little difficult to separate varying $P$ waves from a wandering pacemaker, but in a number of records there was variation of shape and amplitude of the $P$ wave without any change in the $P-R$ or $R-R$ interval. In some this variation was a respiratory variation. In other cases the variation in the $P$ wave was quite striking, namely "pulmonary $P$ waves" present in one record and strikingly absent in another record taken a few hours or days later. This disappearance of "pulmonary $P$ waves" did not always mean improvement. In 7 it was accompanied by deterioration. Altogether, "pulmonary $P$ waves" were recorded at some time in 45 patients.

\section{SHORT P-R INTERVAL}

The short $P-R$ interval has been taken as one less than $0.12 \mathrm{sec}$., with heart rate of 100 a minute or more. There were 12 such cases, but in all the $P-R$ interval was between $0.10 \mathrm{sec}$. and $0.12 \mathrm{sec}$. Five of these were examples of nodal rhythm which have been referred to previously. This left 7 patients with a shortened P-R interval and normal $P$ waves at some time during their illness but in other records normal $P-R$ intervals. Three of these patients also showed marked variation of the $P$ waves.

\section{Prolonged P-R INTERVAL}

This has been taken as a $P-R$ interval of 0.20 sec. or more with a heart rate of 100 a minute or more, and was present in 8 cases. Of the 8,3 were later shown to have coronary artery disease, and 1 was attributed to digitalis overdosage. Another was associated with cortisone withdrawal.

\section{Prolonged Q-T InTERval}

This finding was present in 16 cases. In all these the Q-T interval was $0.34 \mathrm{sec}$. or more, with heart rate of 140 a minute or more. The Q-T was also prolonged at slower heart rates in most of these cases. The common factors were severe illness with hypoxia, and in 12, cardiographic evidence of right ventricular hypertrophy or pulmonary heart disease. Two were interesting because they had a severe exacerbation of asthma, and in these the cardiogram on recovery was normal. Four patients with pulmonary arterial branch occlusion were also in this group. Serum electrolyte balance was normal in all but one of these patients. The findings suggest that acute exacerbation of illness with hypoxia could be responsible for prolongation of the Q-T interval, and this is recorded in some cases before tachycardia or arrhythmia occurred.

\section{Atrial and Ventricular Ectopic Beats}

These were surprisingly frequent, occurring in 40 patients. Fifteen were frequent atrial ectopics, and atrial tachycardia followed in 7 of these. In 13, ventricular ectopic beats were associated with atrial tachycardia, appearing either before the episode or more commonly after it had been controlled. In no case was there a record of the start of the tachycardia, so the part played by an ectopic beat in initiating the abnormal rhythm is not shown. The persistence of multi-focal ventricular ectopic beats carried a poor prognosis.

\section{Discussion}

A review of patients with chronic pulmonary disease has shown that arrhythmias and tachycardias occur in 7 per cent. This proportion will vary with the severity of the illness and the accuracy of the diagnosis. Suppa et al. (1967) found 9.4 per cent in their series of 53 patients with chronic pulmonary heart disease, and their findings agree with ours. Corazza and Pastor (1958) had a frequency of 38.3 per cent in 122 patients with chronic cor pulmonale, but their proportion of very ill patients was high. Transitory atrial arrhythmias were observed in almost one-half of the patients studied by Rochemaure et al. (1967). The inclusion of unsuspected cases of coronary heart disease may increase the frequency of atrial arrhythmias and tachycardias. In a previous such series with both conditions present our incidence was 6 per cent (Rees et al., 1964). Tachycardias occur with obstructive pulmonary hypertension, pulmonary fibrosis, and pulmonary thromboembolism, particularly where there is an exacerbation of hypoxia. The more frequent and careful the cardiographic study the more commonly are abnormalities of rhythm and rate identified.

The causation of arrhythmias and tachycardias has been discussed by Goldberg et al. (1960), Bellet (1963), and Suppa et al. (1967). All possible factors have been suggested. Bellet (1963) puts prolonged right heart strain, anoxia, infection with coughing initiating vagal reflexes, and electrolyte imbalance as probable causes. Goldberg et al. (1960) have much the same view but suggest that paroxysmal atrial tachycardia with AV block is frequent and is related to digitalis overdosage with reduced cellular potassium. In this study this condition was infrequent. Suppa et al. (1967) state that the more 
severe disorders of cardiac rhythm bear some relation to the right heart failure and respiratory insufficiency with hypercapnia. Rochemaure et al. (1967) noted that all attacks of atrial arrhythmias occurred during exacerbation of respiratory insufficiency with hypercapnia. Sinus tachycardia is common in all series, but there is no direct relation to any one factor.

In an earlier study, Thomas, Cotes, and Pisa (1963) showed that the $P$ wave abnormalities and atrial tachycardias occurred in those patients with the highest pulmonary pressures, the average of these being $\mathrm{P} \overline{\mathrm{PA}} 37.44 \mathrm{~mm}$. $\mathrm{Hg}$, whereas it was $22.9 \mathrm{~mm}$. $\mathrm{Hg}$ in chronic lung disease with normal $P$ waves. In this series arrhythmias and tachycardias were associated in many cases with cardiographic evidence of pulmonary hypertension, but there were some with pulmonary disease only and exacerbation of chest infection, respiratory failure, or obstruction of a branch of the pulmonary arterial tree. These happenings would all produce a further increase in pulmonary arterial pressure, and it is suggested that this is a common factor in precipitating arrhythmia and tachycardia. Bellet (1963) remarks on the appearance of cardiographic changes including arrhythmias after exercise in patients with cor pulmonale. It has been shown that the resting heart rate and the increment in heart rate on exercise is higher the more severe the pulmonary disease (Gilson and Hugh-Jones, 1955). Both exercise and intercurrent chest infection would produce increases in pulmonary arterial pressure and in heart rate in these patients, and either could predispose to arrhythmia.

A factor which relates to the change in form of the $\mathrm{P}$ waves is increase in intrathoracic pressure. We noted that positive pressure breathing could produce a change from normal to tall pointed $P$ waves in patients with pulmonary hypertension. Such alterations occur without assisted respiration in patients with severe ventilatory obstruction. Such $P$ wave changes have been seen by others, for example in a recording of intermittent positive pressure breathing shown by Bristow, Morris, and Kloster (1966). Rochemaure et al. (1967) say that accentuation of the signs of right atrial hypertrophy is sometimes recorded under assisted ventilation, but they and Bristow et al. refer to this as a haemodynamic change. $P$ wave abnormalities of form and duration are frequent in our series, and a change from "pulmonary $P$ waves" to more normal form did not always signify improvement. Scherf and Cohen (1964) state that wandering of the pacemaker is due to changes of tonus of the autonomic nervous system, which are more common in the elderly.
Some of our patients are elderly, but exacerbation of the pulmonary disease is still the more likely explanation.

There was no relation in this series with digitalis effect or low serum potassium because safeguards against these were present. Arrhythmias and tachycardias occurred with normal serum electrolyte levels, but in 6 cases additional potassium was given for another reason, with a resultant change of $P$ waves towards their previous normal form, but this did not mean clinical improvement in every case. Such normalization of $\mathbf{P}$ waves after potassium administration has been noted by others, and may be explained by the recent suggestion of Mills, Wilson, and Craig (1968) that total exchangeable potassium is sometimes very depleted with a normal serum potassium. Changing form of the $P$ wave, shortening of the $P-R$ interval, or prolongation of the $Q-T$ interval appeared, one or more, in patients who later developed arrhythmias. The appearance of these is usually associated with worsening of the ventilatory capacity and hypoxia.

Examples of atrial flutter and atrial fibrillation are shown in pure pulmonary heart disease, but atrial tachycardia is the most common finding in most series (Suppa et al., 1967). As in other series (Rees et al., 1964), arrhythmias, and particularly atrial fibrillation, are more frequent when coronary heart disease and pulmonary heart disease occur together.

Of considerable interest was the finding of pulmonary arterial occlusion in 11 of these patients and the likely association of this with an episode of tachycardia or arrhythmia. In 2 the occlusion followed leg vein thrombosis, in one the embolus originated in the right atrium but in the others the thrombus may well have formed in situ. Brun et al. (1965) have described similar cases, but they suggested the right atrium or ventricle as the source of the embolism. It is probable in this series that pulmonary arterial thrombosis commonly occurs in situ because of the inclusion of patients with pneumoconiosis who are prone to thrombosis of the pulmonary arterial tree. Such occlusions increase the pulmonary hypertension and the hypoxia and so precipitate tachycardia.

The occurrence of most forms of arrhythmias and tachycardias was related to a poor prognosis, with death likely within two years, but there were some striking exceptions. Asthma produced 3 examples of atrial tachycardia, 3 of varying P waves, and 6 with ST-T changes in sinus tachycardia with additional ectopic beats. The prognosis here was good if the asthma improved, presumably because of a return to normal of the pulmonary artery pressure 
and the arterial oxygen saturation. In chronic bronchitis the persistence of severe ventilatory disability and pulmonary hypertension caused a liability to recurrence of arrhythmia and tachycardia and a poor prognosis.

Management is directed to the rapid control of infection, improvement in ventilation and hypoxia, and so some reduction in the pulmonary arterial pressure. Such measures frequently resulted in spontaneous reversion to normal rhythm. Digitalis has been used, but with care, because of the susceptibility to paroxysmal atrial tachycardia with block. Procainamide has been of value in a number of cases, and more recently antazoline has been used successfully, particularly in the control of ectopic beats. It is important to ensure that the serum potassium is normal, and even in some cases with an apparent normal serum potassium, improvement occurred in the arrhythmia and tachycardia when extra potassium was given. The recent introduction of anti-arrhythmic agents with beta-adrenergic blocking effect but without a bronchoconstricting effect may prove helpful in the management of some of the more difficult arrhythmias, but only in addition to the fundamental improvement of ventilation and hypoxia.

\section{SUMMARY}

Arrhythmias and tachycardias occur in 7 per cent of patients with severe chronic lung disease. They are most common in those with cardiographic patterns of pulmonary heart disease or right ventricular hypertrophy. Frequent precipitating factors are intercurrent chest infections, but respiratory failure and thromboembolic occlusions of pulmonary artery branches can be important factors. An increase in pulmonary hypertension is thought to be the basic common factor in these patients. Neither digitalis overdosage nor low serum potassium levels were evident in this series.

Cardiographic signs indicative of possible arrhythmias or tachycardias are changing form of the $P$ waves, shortening of the $P-R$ interval, or prolongation of the Q-T interval. Atrial tachycardia, nodal rhythm, and wandering pacemaker were common findings. Atrial and ventricular ectopic beats persisting after correction of arrhythmia and tachycardia suggested a worsening prognosis. Most patients experiencing a serious arrhythmia or tachycardia were dead in two years, but asthma was a notable exception. The presence of unsuspected coronary heart disease was responsible for some rhythm disorders, especially atrial fibrillation. A basic requirement for the control of the disordered heart beat was rapid improvement in ventilation and correction of hypoxia.

We wish to express our gratitude to our colleagues Dr. Byron Evans and Dr. D. A. Williams, and especially to Dr. J. D. Ball of the Miners' Chest Diseases Centre, for permission to include their patients in this study. We acknowledge with gratitude the help received from the Cardiological Technicians. We thank Dr. T. Parry and Dr. F. K. Storring for the pathology necropsy reports.

\section{REFERENCES}

Bellet, S. (1963). Clinical Disorders of the Heart Beat, 2nd ed., p. 695 . Lea and Febiger, Philadelphia.

Bristow, J. D., Morris, J. F., and Kloster, F. E. (1966). Hemodynamics of cor pulmonale. Progr. cardiovasc. Dis., 9, 239.

Brun, J., Gardère, J., Kalb, J. C., and Pozzetto, H. (1965). Thromboses cardio-pulmonaires et leur traitement par les fibrinolytiques au cours des syndromes de détresse respiratoire compliquant bronchite chronique et emphysème. Poumon, 21, 327.

Corazza, L. J., and Pastor, B. H. (1958). Cardiac arrhythmias in chronic cor pulmonale. New Engl. f. Med., 259,862 .

Gilson, J. C., and Hugh-Jones, P. (1955). Lung function in coalworkers' pneumoconiosis. Spec. Rep. Ser. med. Res. Coun. (Lond.), No. 290, p. 93.

Goldberg, L. M., Bristow, J. D., Parker, B. M., and Ritzmann, L. W. (1960). Paroxysmal tachycardia with atrioventricular block. Its frequent association with chronic pulmonary disease. Circulation, 21, 499.

Mills, I. H., Wilson, R. J., and Craig, G. M. (1968). The mechanisms of sodium retention in cor pulmonale; the role of potassium. Paper read at Association of Physicians of Great Britain and Ireland, April 1968.

Rees, H. A., Thomas, A. J., and Rossiter, C. (1964). The recognition of coronary heart disease in the presence of pulmonary disease. Brit. Heart f., 26, 233.

Rochemaure, J., Bignon, J., Khoury, F., and Brouet, G. (1967). Confrontations anatomo-électrocardiographiques chez 50 patients atteints de broncho-pneumopathies chroniques. Arch. Mal Coeur, 60, 1615.

Scherf, D., and Cohen, J. (1964). The Atrioventricular Node and Selected Cardiac Arrhythmias, p. 141. Grune and Stratton, New York and London.

Suppa, G., Martini, M., and Gazzi, A. (1967). Le aritmie nel cuore polmonare cronico. Mal. cardiovasc., 8, 225.

Thomas, A. J., Cotes, J. E., and Pisa, Z. (1963). Pulmonary hypertension in patients with chronic lung disease. In Proc. IV World Congr. Cardiol., Mexico, 1962, Vol. I.B., p. 180. 